\title{
Germanica
}

\section{Théâtralisations radiophoniques de poèmes}

Elian Beaufils: Lyrische Hörspiel-Theatralität

Eliane Beaufils: Lyrical theatricalisation in the radio

\section{Eliane Beaufils}

\section{CpenEdition}

\section{Journals}

Édition électronique

URL : http://journals.openedition.org/germanica/7140

DOI : 10.4000/germanica. 7140

ISSN : 2107-0784

Éditeur

Université de Lille

Édition imprimée

Date de publication : 26 juin 2019

Pagination : 183-198

ISBN : 978-2-913857-42-1

ISSN : 0984-2632

Référence électronique

Eliane Beaufils, «Théâtralisations radiophoniques de poèmes », Germanica [En ligne], 64 | 2e trimestre 2019, mis en ligne le 01 janvier 2021, consulté le 27 février 2021. URL : http://journals.openedition.org/ germanica/7140 ; DOI : https://doi.org/10.4000/germanica.7140

(c) Tous droits réservés 


\title{
Théâtralisations radiophoniques de poèmes
}

\author{
Eliane Beaufils \\ Université Paris 8
}

La poésie a longtemps été la reine des scènes théâtrales, Brecht y recourait encore par l'entremise de ses songs et autres rimes. Après la guerre cependant et avec l'avènement du Regietheater s'est creusée la distance prise avec le texte et les énonciations qui lui étaient associées, comme le fait remarquer Helga Finter. La chercheuse explique que cette distance résulte en premier lieu de l'instrumentalisation de la parole poétique par le nazisme :

D’une part, la méfiance des hommes de théâtre vis-à-vis de voix esthétiquement très travaillées est due au caractère lisse et creux d'un style de langage rhétorique qui s'est perpétué jusqu’à l'époque Adenauer [...]. Mais des styles de voix caractéristiques du théâtre allemand ont par surcroît été subtilisés par Hitler et Joseph Goebbels, et de façon tout à fait caricaturale ${ }^{1}$.

Les pièces radiophoniques après-guerre renoncent également à tout pathos poétique. La poésie orale a néanmoins subsisté sous forme de

1. - Helga Finter, « Mit den Ohren sprechen », in : Heiner Goebbels, Nikolaus Müller-Schöll (dir.), Heiner Müller sprechen, Berlin, Theater der Zeit, 2009, p. 62-72, ici p. 67. «Einerseits ist für dieses Misstrauen der Theatermacher gegenüber der kunstvollen Stimme die Glätte und der hohle Schönklang eines Sprechstils verantwortlich, der bis in die Adenauer-Zeit fortwirkte [...]. Mit Hitler und Joseph Goebbels waren auch - zur Karikatur verkommen - charakteristische Stimmstile des deutschen Theaters entwendet worden. » 
Hörspiele spécifiques, notamment avec Gerhard Rühm et Ernst Jandl, avant de connaître un nouvel essor grâce aux poetry slams. Des présentations de textes poétiques beaucoup plus quotidiens et moins formels se répandent désormais sur les ondes, ce qui semble affecter la façon même dont on peut reconnaître le caractère poétique du dire. J'aimerais de ce fait me pencher sur certaines de ces nouvelles théâtralisations sonores du lyrisme en rupture avec les anciennes formes. Ces Hörspiele ne se prétendent pas toujours poétiques au demeurant, mais la théâtralisation sonore contribue à un " dire poétique » qu'il importe de circonscrire. Afin de caractériser certains modes de théâtralisation sonore et le dire poétique qu'ils supportent, j'étudierai en particulier la pièce Ewig ohne Dauer, de Christian Uetz. Le rythme et l'intonation y jouent un rôle essentiel. L'énonciation poétique semble également aller de pair avec une expérience contée telle que l'envisage Walter Benjamin. Or il est intéressant d'observer que cette transmission d'expérience poétique peut également se faire tout en portant sur des contenus beaucoup plus politiques et prosaïques, grâce à des dynamiques sonores semblables. C'est pourquoi j'évoquerai deux autres pièces, Urban Prayers de Björn Bicker, et Europas Längster Sommer de Maxi Obexer, pour clore cette réflexion sur l'espace radiophonique poétique.

\section{Ewig ohne Dauer : entre spéculation et performativité}

Avant de montrer comment Ewig ohne Dauer ${ }^{2}$ se théâtralise à la radio, il convient de revenir sur le poème lui-même. La difficulté à distinguer la forme du contenu a toujours été soulignée vis-à-vis de la poésie, mais il semble que cela ait ici une incidence particulière.

Le point de départ du poème est la Sehnsucht, notion poétique s'il en est du désir mélancolique. Dès les premières phrases, celle-ci est ramenée à un objet : le présent sans présent. Le poème affirme que ce que l'on recherche est l'esprit présent à lui-même (Geistesgegenwart). Or cette présence d'esprit est un «présent sans présent» : c'est l'espace d'un présent pensé, présent à lui-même, qui échapperait au présent par une forme de présence dégagée de l'action. Le présent sans présent serait visé, présent, sans jamais pouvoir être l'objet d'une saisie, $a$ fortiori d'une maîtrise. De par son impossible saisie, il nourrirait le désir, attiserait la pensée, serait désir de plaisir infini, lui-même sans

2. - Ewig ohne Dauer est une pièce du poète suisse Christian Uetz, produite par WDR 3, en 2004. Il s'agit de la seule pièce radiophonique de l'auteur à notre connaissance. Les acteurs sont Christian Uetz, Andreas Grothgar, Bettina Engelhardt. Bert Wrede a effectué la composition sonore. Ulrich Lampen est le responsable de la mise en scène. La pièce est disponible en podcast. Voir https://wwwl.wdr.de/mediathek/ audio/wdr3/wdr3-hoerspiel/audio-ewig-ohne-dauer-102.html, consulté le 25 mai 2019. Par la suite, abrégé par Uetz suivi de l'indication des minutes. 
fin. Ewig ohne Dauer décrit ainsi une pensée qui représente à la fois un enjeu spéculatif et l'objet d'une quête perpétuelle. Cette recherche semble également se poursuivre hic et nunc, car le poème tend à s'inscrire dans ce présent sans présent qu'il cherche à décrire. Il y a, presqu'à chaque pas, une dimension performative de ce dire qui cherche à s'autoaccomplir. Si ce n'est que le poème cherche à le faire sans l'expliciter, sans ramener métaréflexivement la recherche au poème lui-même. La parole poétique reste dans une sorte d'état de suspension vers ce qu'elle saisit sans pouvoir le saisir ni l'accomplir, en un mouvement sans fin.

Elle est par ailleurs souvent prise dans les mouvements dialectiques ou sophistiques propres à toute pensée active : le poète souligne par exemple qu'on n'a pas le droit de préjuger qu'on comprend l'autre, ni de préjuger de quelque droit sur l'autre ; mais aimer c'est penser comprendre l'autre, même sans parole, comment donc accepter de ne pas vouloir comprendre l'autre ? À un autre moment sont listés les pièges de la preuve, de la volonté d'être, de représenter pour l'autre en faisant fi du « rien » qui devrait contenter l'amour. Le texte communique alors la processualité d'une pensée en train de se faire et de se défaire. L'oralité de la poésie a moins à voir ici avec le jeu sur les sonorités et le rythme, qu'avec la spéculation et le rythme d'une réflexion vivante (bien que la répétition des termes et la variation des locutions qui se fondent sur eux jouent indubitablement un rôle rythmique).

Le langage est inéluctablement thématisé comme le vecteur, le médiateur, l'impulsion de ces mouvements de pensée : " absence présente et présence absente $»^{3}$, il est ce par quoi une chose vient à exister, et une chose ne vient à exister que s'il existe des mots préalables pour la dire. Le poète et penseur Henri Meschonnic raille les poètes qui célèbrent la puissance ou impuissance de nomination du langage ${ }^{4}$, mais ici le langage est une puissance d'existence paradoxale dont l'impuissance, la fragilité, sont affirmées dans le temps même où se développe la puissance du désir de pensée. Le poème devient « encre de la mélancolie » comme l'exprime Jean Starobinski ${ }^{5}$ : dans son ouvrage éponyme, l'auteur n'opère pas seulement une généalogie littéraire du sentiment mélancolique (forme dépressive paradoxalement source de création comme le Hörspiel l'exemplifie également) mais souligne l'importance des vocables exprimant « mélancolie » et « nostalgie » pour les écrivains (Sehnsucht en allemand, saudade en portugais, la pensée du désir et de la mélancolie prend des traits différents dans chaque langue).

3. - « anwesende Abwesenheit und abwesende Anwesenheit» (Uetz, 2'19).

4. - Henri Meschonnic, Célébration de la poésie, Lagrasse, Verdier, 2001, p. 150 par exemple.

5. — Jean Starobinski, L'Encre de la mélancolie, Paris, Seuil, 2012. 
Comme le flux de la pensée ne cesse de se développer de manière dialogique et dialectique, apportant des réponses qui se dérobent aussitôt pour faire place à des objections ou des perspectives déplacées, cela rejaillit sur la dialoguicité interne du poème. De même que dans moultes poésies, le « je » s'adresse à un «tu », mais ce « tu » est ici démultiplié : il est souvent susceptible de renvoyer aussi bien à la personne aimée, à soi-même, à la Sehnsucht, voire à l'amour : " denn dich habe ich gesucht - und drum nicht gefunden/ durch dich kann ich meine Liebe finden/ durch dich habe ich Gegenwart erfahren/ dein Begreifen begreift dich » (Uetz, 15'36). Le mouvement du langage rejoint en définitive celui de l'amour, et celui du désir, il se dessine une convergence polyforme des motions de la pensée.

Ainsi la pluralité des voix est interne au poème, avant même d'être accentuée par celle des trois acteurs à la radio. Cette pluralité met plus largement l'accent sur le fait qu'il n'y a pas de narrateur, ni même de je lyrique homogène ; qu'il n'y a pas de conscience claire possible qui serait un point d'ancrage de la réflexion. Il n'existe pas a priori de point de fuite vers lequel convergeraient toutes les réflexions, qui trouveraient, à défaut d'un sens univoque, une sorte d'aboutissement dans un « je lyrique » sage, capable de nouer les choses. Cependant, maintenir le dialogue de la pensée avec elle-même, qui se répond et se dédouble, est sans doute la condition même pour devenir sujet, saisir un « je » à défaut de se saisir. Pour le philosophe Jean-Luc Nancy, « $[u] n$ sujet se sent, c'est sa propriété et sa définition. C'est-à-dire qu'il s'entend, se voit [...], se pense [...], s'approche et s'éloigne de soi, et toujours ainsi se sent « sentir » un soi qui s'échappe ou qui se retranche, autant qu'il retentit ailleurs $»^{6}$. De cette façon, la pensée qui ne peut s'accomplir, révèle le sujet à lui-même. Et de cette façon, le poème renoue avec un "rythme » tel que l'entend Meschonnic, le rythme d'une pensée qui exprime un mouvement de subjectivation quoiqu'elle « n'aboutisse » pas ${ }^{7}$.

Cette pensée en suspension qui ne cesse de revenir sur elle-même est proche du rêve. Le poète martèle en vérité l'importance du rêve, nécessaire à la pensée, à l'existence de l'autre, des sentiments, au faire sens et au « faire réel »: sans rêve, pas de réalité. Voilà qui va bien au-delà ou en-deçà des fictions auxquelles l'esprit selon Nietzsche est tenu d'avoir recours pour penser et ordonner le réel : le rêve est ici associé au suspens de la pensée, à sa direction, à sa dérive vers l'objet de pensée qu'elle ne peut jamais atteindre tout à fait. Il est vu comme prolongement ou complé-

6. - Jean-Luc Nancy, À l'écoute, Paris, Galilée, 2002, p. 25.

7. - Le rythme revêt de ce fait une importance cruciale dans la pensée de Meschonnic si bien qu'il a donné lieu à son maître ouvrage, Critique du rythme. Anthropologie historique du langage, Paris, Verdier, 1982. 
ment de la pensée spéculative. Ce n'est pas la plongée dans les choses qui permettrait l'investissement de subjectivité - sous couvert d'action, ou de réalisme. Au contraire, le réalisme, « les réalités anéantissent la vie »8.

Par ces invocations du rêve, par ces circonvolutions de la réflexion, l'écoute poétique à son tour est appelée à être tant spéculative qu'onirique. L'auditeur de la pièce ne peut précéder les paroles, il doit accepter d'être lui-même suspendu à la recherche poématique, à la réflexion sur le présent sans présent qui se dérobe à lui-même et qui lui dévoile son désir. Le dire poétique a en soi un effet performatif de suspension, qui catalyse par ailleurs le désir (d'écoute). Or quand la pensée cherche ainsi à saisir et à se saisir, à s'éprendre d'objets tout en revenant sur elle-même, se développe une théâtralité de la pensée, une autoréflexion. Cet effet est accentué quand le texte reprend les mêmes racines étymologiques : « [Die Sehnsucht] ist nicht zu halten. Und ich halte haltlos daran fest ». Ou quand il enfile les adjectifs : « Sehnsucht, als die Form der Verliebtheit in die Gegenwart, die heftigste, die schmerzlichste, die brennendste, die zärtlichste, die allgemeinste ». Ou quand un principe de contradiction déjoue les clichés : « du bist nicht mein/ ich bin nicht dein/ und dessen bist du dir nicht gewiss ${ }^{9}$. Ces négations vont à l'encontre des attentes, ou du moins des discours courants, et provoquent des sortes de retournement de la pensée sur elle-même. Certaines expressions renouent par ailleurs avec une poésie d'images autant que de spéculation : « Krank sein und im Bett liegen/ nicht krank sein und im Bett liegen/ die Krankheit sein und im Bett liegen lassen/mit der Krankheit des Seins im Nichts des Bettes liegen und gesund sein/ im Bett des Nicht-Seins liegen » (Uetz, 5'19-5'34). Lister des phrases reposant sur des variations parfois minimes était un procédé très cher à Gertrude Stein, qui avait à cœur de se concentrer sur l'instant présent, pour ne pas être toujours en avant ou en arrière des sentiments mis en scène, et qui aimait se concentrer sur la différence dans la ressemblance $^{10}$. Le Hör - spiel sait jouer avec les mots et les imaginaires.

Le texte éminemment poétique par son objet, son autoréférentialité, l'adresse à un « tu » et ses caractéristiques formelles, est donc aussi théâtral et performatif. Il semblerait que le poète développe sa poésie comme Hörspiel, pièce radiophonique, parce que sa pensée s'y performe : le rapport à l'objet du discours tend à se confondre avec la forme du discours qui autoaccomplit d'une certaine façon ce dont elle

8. — «Realitäten vernichten das Leben » (Uetz, 8’08).

9. - Cf. respectivement Uetz, 1'22, 0'21 (entre autres) et 20'31. Ces derniers vers reprennent en l'occurrence le poème bien connu du haut Moyen-Âge « du bist mîn, ich bin dîn, des solt du gewis sîn » qui repose sur une appartenance et une réciprocité auxquelles renonce Uetz.

10. - Voir notamment Gertrude Stein, Lectures in America, Boston, Beacon Press, 1935. 
est en recherche, le désir, l'amour, la Sehnsucht. J'essaierai de montrer désormais que par bien des aspects, le caractère oral et sonore de la présentation à la radio en accroît la théâtralité et la performativité.

\section{Comment le poème oral parle à l'auditeur}

A priori, l'oralisation du poème pourrait pourtant sembler porter préjudice à sa réception. Les sociétés occidentales ne sont plus des sociétés de culture orale, a fortiori rituelles, la poésie orale est donc relativement inhabituelle. Par ailleurs, le rythme des paroles peut paraître artificiel, au sens où ce sont des acteurs qui s'en emparent et que l'auditeur ne saurait, comme le fait le lecteur de poèmes, s'engager soi-même dans les brèches du texte. Il ne peut par conséquent se sentir parent de la voix poétique qui s'y déplie. Il ne peut pratiquer cette activité de ré-énonciation qui est au cœur de l'expérience de la lecture poétique selon Eric Benoit :

[...] la poésie est un genre littéraire qui donne à la parole écrite la capacité d'être disponible à une perpétuelle ré-énonciation, une perpétuelle réactualisation comme événement singulier dans la profération. Le lecteur perçoit la voix qui dit « je » dans le poème et se la réapproprie par empathie. Par la voix, la parole du poème est événement, et peut redevenir événement toujours nouveau dans la lecture comme expérience existentielle ${ }^{11}$.

Mais on pourrait rétorquer que la forme éminemment dialogique du poème, la réflexion vivante qui s'y déplie, la quête ostensible de subjectivation qui le sous-tend, invitent l'auditeur à participer à cette réflexion permanente qui questionne et répond, et au rythme qui soustend ces méandres de la réflexion. L'auditeur est d'autant plus appelé à acquiescer aux réponses ou prolonger la réflexion, à participer à ce mouvement de saisie et dessaisie de la pensée, qu'il est suspendu à l'écoute. Il est pris dans les rets des « tu » du poème, l'oralité accroît la performativité de la suspension et la dialoguicité, les trois voix multiplient les apostrophes. Par ailleurs, la sollicitation du rêve auquel est dévolu un rôle clé dans la pensée fait également appel à l'imaginaire oral de l'auditeur, « tendu vers $1[\mathrm{es}]$ voix » qui sont toutes en sus associées à un imaginaire corporel ${ }^{12}$. L'appel à l'auditeur repose par ailleurs sur la théâtralisation spécifiquement radiophonique et non seulement sonore, qui contribue à faire de l'écoute un événement, et permet peutêtre une « expérience existentielle».

11. - Éric Benoit, Dynamiques de la voix poétique, Paris, Garnier, 2016, p. 56.

12. - Sandrine Le Pors, Le Théâtre des voix. À l'écoute du personnage et des écritures contemporaines, Rennes, PUR, 2011, p. 119. 
De fait, comme toute pièce radiophonique, celle-ci est en premier lieu théâtrale par son support. Qui dit radio et podcast, dit possibilité d'écoute en tous lieux, et presque en toute situation. Ce qui est dit s'inscrit dans un environnement, voire une action. Il est donc rattaché à des occupations quotidiennes lesquelles sont mises à distance par les voix radiophoniques et deviennent un théâtre du quotidien. Comme le quotidien suit son cours sans se soucier des voix poétiques, se produit un heurt des temporalités. Alors que les voix des comédiens contribuent à une abstraction du cadre de vie, et à un suspens du temps, l'auditeur peut avoir l'impression qu'elles mettent la vie en perspective autrement et en dévoilent des dimensions irréfléchies : ne décrivent-elles pas le cœur du désir, donc le cœur de la vie ? Il existe en somme un écho situationnel des propositions poétiques dans le quotidien de l'auditeur. Les voix s'inscrivent malgré tout dans un cadre qui est a priori détaché d'elles. Cela rend la spéculation encore plus vivante.

Cet écho situationnel est susceptible d'être d'autant plus important qu'une tension se crée avec la « présence d'esprit » conjurée par le poème, cet esprit présent qui va de pair avec le « présent sans présent » vers lequel tendent les voix : car ce présent doit s'extraire du quotidien précisément. L'évocation du présent sans présent tend donc à aiguiser l'attention de l'auditeur, d'autant qu'elle le conjure comme un état que chacun viserait. Par surcroit, le rythme relativement rapide de l'énonciation, l'enchaînement de propositions complexes, rendues plus complexes encore par les jeux de mots et de racines étymologiques, les nuances et les contradictions, exigent de l'auditeur une attention de tous les instants. De ce fait, l'attention et le désir mélancolique évoqués de manière théorique sont en partie réalisés performativement par l'auditeur comme attention et désir de compréhension.

Les voix radiophoniques mettent d'autre part l'auditeur sur la voie du présent sans présent grâce à des pauses ménagées régulièrement : au flot de phrases succèdent en effet des plages de silence où l'auditeur peut laisser résonner les paroles entendues. Des accords électroniques meublent les silences. Ils sont assez neutres pour ne pas donner lieu à des mélodies, a fortiori à des musiques narratives ou évocatrices. Ils soutiennent ainsi l'activité subjective, voire autopoïétique, de l'auditeur ; contribuent à la fois à l'inscription dans le quotidien et à l'intégration subjective des contenus. D'autres dimensions contribuent à rendre la parole " vive ${ }^{13}$, et à instaurer une tension avec le caractère écrit des paroles, l'artifice des voix enregistrées, rythmées, et jouées. Si l'auditeur n'a pas la possibilité de s'immerger dans les mots comme le lecteur, l'altérité des voix, leur pluralité accentuent encore la

13. - Selon l'expression de Platon dans Phèdre, reprise par Jacques Derrida dans De la grammatologie, Paris, Seuil, 1966. 
dialoguicité interne au poème, les contradictions ou les nuances apportées. Au lieu de recevoir simplement différentes inflexions, les phrases reçoivent différentes voix ; la réflexion en est encore plus dynamique.

Par le développement d'une réflexion vivante et dynamique jointe à l'altérité, l'oralité contribue à faire de l'écoute une expérience de pensée. Le poème devient l'espace d'une transmission d'expérience, et les dires acquièrent une dimension narrative. Ewig ohne Dauer réinvente en somme à sa manière une façon de conter et de transmettre des expériences. Walter Benjamin appelait en effet de ses vœux des conteurs qui ne se contentent pas d'être des passeurs d'informations, mais qui, par leurs intonations, le choix des termes, le prisme subjectif de la narration montrent à quel point le sens d'une histoire ne peut être épuisé par un compte-rendu factuel. Le sens est redevable de l'engagement de chacun, si bien qu'il y a transmission suivant une double modalité chez le conteur : il montre le sens qu'on a attaché à des histoires et il montre en même temps la part subjective, dynamique du sens qui ne s'épuise pas dans la rationalité. Par-delà des attitudes vis-à-vis d'actions et de la vie, il transmet une attitude vis-à-vis des faits, de ce qui fait événement, et de la part opaque de ce qui fait événement ${ }^{14}$. Le poème Ewig ohne Dauer est d'autant plus susceptible d'être conteur qu'il relaie l'expérience d'un poète, Christian Uetz - impression confortée par le fait que l'un des locuteurs est l'auteur en personne.

Surtout, le poème soumet à l'appréciation de l'auditeur un langage qui contraste furieusement avec celui qu'il est accoutumé à entendre dans les médias. Cette langue ne peut guère être rattachée au «théâtre d'affects ", par lequel le philosophe Byung-Chul Han comprend non seulement les spectacles présentés dans les théâtres, mais aussi les mises en scène de la vie dans les réseaux sociaux, le caractère un peu hystérique de maintes modérations, ou les intrigues mélodramatiques des séries télévisées : dans tous les médias, on cherche à " décharger des affects $\rangle^{15}$ et à faire consommer des émotions. Le dire du poème ne relève pas non plus d'une énonciation d'universitaire ou de journaliste, qui cherche à montrer son statut et/ou à rendre manifeste sa rationalité par la qualité formelle de son langage, de son argumentation et par ses intonations pondérées. La pièce radiophonique rompt tout à fait avec ces énonciations qui, sans être toujours standardisées, répondent à des attentes ; elle produit une disruption par rapport à l'environnement médiatique et à nos habitudes. Elle instaure un autre temps et un autre mode - bien qu'il convienne sans doute de ne pas exagérer sa portée

14. - Walter Benjamin, «Le Conteur. Réflexions sur l'œuvre de Nicolas Leskov », trad. Pierre Rusch, in : Id., Euvres III, Paris, Gallimard, 2000, p. 114-151.

15. - Byung-Chul Han, Psychopolitik. Neoliberalismus und die neuen Machttechniken, Frankfurt a.M., Fischer, 2015, p. 63 : «Affekte entledigen ». 
hétérotopique, comme le souligne Nikolaus Müller-Schöll vis-à-vis des îlots de théâtre en général ${ }^{16}$. Mais elle est en rupture avec l'économie attentionnelle dominante, et promeut une expérience auditive et spéculative qui fait écho à celle que représente le poème.

Pièce réflexive sur le désir et l'amour, elle n'appelle pas à une empathie. Pour Byung-Chul Han cependant, le sentiment (Gefühl), à la différence de l'émotion et de l'affect, est doté d'une « longueur narrative »17. Il est doté d'une durée, et « ouvre un espace $»^{18}$. Lors de la présentation radiophonique, l'auditeur est suspendu à un temps et à un espace concret-réflexif, reçoit des propositions à qualité narrative, et est invité à prolonger l'expérience de pensée. La pièce rejoint ainsi en quelque sorte le propre du sentiment. Sans faire appel à l'empathie, la théâtralisation permet de rendre perceptible la narrativité ou dramaticité propre au sentiment, d'autant que l'auteur fonde amour et désir en premier lieu sur la conscience et la pensée : rappelons que pour lui, l'amour n'existe guère sans les mots de l'amour et les retours réflexifs sur lui. Narrativité et procédés disruptifs usuellement rapportés à la poésie sont ici complémentaires : on pourrait dire que " la narration devient le moyen de mise en place d'un jeu de ruptures et de discontinuités temporelles $»^{19}$ autant que le poème devient le moyen d'une transmission contée. Une des « transmissions » secondaires pourrait résider par ailleurs dans un mode de subjectivation : en faisant écho à sa subjectivité, en s'ancrant dans un environnement, voire dans des situations, et en promouvant un retour sur sa propre constitution en matière de pensée et d'amour, les mots initient une expérience chez l'auditeur; ils initient plus loin une subjectivation qui fait écho à celle qui est à l'œuvre dans le poème et qui contribue sans doute aussi au désir de ré-énonciation ${ }^{20}$.

Enfin, par l'oralité, la multiplication des voix et des adresses (« tu »), l'altérité de la pièce radiophonique n'accentue pas seulement la dimension

16. - Le chercheur francfortois refuse de considérer tous les spectacles plus lents et contemplatifs d'hétérotopies indiciant un autre mode d'être, au prétexte qu'ils « combattraient » la rapidité ambiante. Voir N. Müller-Schöll, «Wie denkt Theater ? Zur Politik der Darstellung nach dem Fall », in: Artur Pelka, Stefan Tigges (dir.), Das Drama nach dem Drama. Verwandlungen dramatischer Formen in Deutschland seit 1945, Bielefeld, transcript, 2011, p. 357-372, ici p. 358.

17. - Byung-Chul Han, op. cit., p. 60 : « narrative Länge ».

18. - Ibid., « öffnet einen Raum».

19. - Clémentine Hougue, « Mises en fiction de la mémoire dans la littérature et les arts du monde anglophone ", Acta fabula, vol. 7, n 4, Août-Septembre 2006, http:// www.fabula.org/acta/document1479.php, consulté le 02 mai 2019.

20. - Selon Meschonnic, le poème est une forme tellement subjectivante qu'il va jusqu'à dire qu'il n'y a pas de sujet du poème, mais que le poème est toujours un " poème-sujet »: « le sujet du poème. Ce n'est pas l'individu, l'auteur. C'est l'activité même de subjectivation d'un discours, d'une pratique ». Voir H. Meschonnic, Célébrations de la poésie, op.cit., p. 43. 
intersubjective de la rencontre poétique. Car tout ce qui est dit à la radio est situé dans l'espace du commun, au-delà de deux subjectivités. La radiophonie promeut un échange « transsubjectif » davantage qu'intersubjectif. L'échange transsubjectif rejoint l'être-ensemble tel que le conçoit Jean-Luc Nancy : un mouvement de sens en commun, où chacun prend sa part de près ou de loin, et où il faut comprendre le sens en tous sens ${ }^{21}$.

La dimension sensible de la langue parlée contribue quant à elle à affecter la pensée. L'affectation est liée à des variables bien connues : le fait que les oreilles ne peuvent être closes, qu'elles constituent le premier sens développé par le fœtus, que l'empreinte d'une voix est plus singulière encore que ne le sont les empreintes digitales. Mais elle ne constitue pas seulement un phénomène passif et intuitif. Selon Hartmut Rosa, la résonance en effet est un phénomène essentiel à la mise en relation de soi avec autrui, c'est une forme de rapport au monde qui se constitue à partir d'affectation et d'émotion, d'intérêts qu'on développe pour eux-mêmes et d'une attente d'auto-efficience, liés à ce que le sujet et le monde objectif se touchent réciproquement et se transforment par là. Pour le sociologue, la résonance n'est pas une relation d'écho mais de réponse ; elle présuppose que les deux parties parlent chacune de leur propre voix, et cela ne serait possible que là où des valorisations fortes seraient en jeu. La résonance ne représente donc pas pour lui un état émotionnel mais un mode relationnel. C'est ainsi, dit-il, que nous pouvons aimer des histoires tristes ${ }^{22}$. La résonance sonore est en outre beaucoup plus palpable que l'écho à la lecture ; elle est rencontre consciente d'une altérité, non prolongement de soi.

Ewig ohne Dauer permet ainsi d'identifier les dynamiques théâtrales qui traversent la présentation sonore de textes poétiques : l'inscription situationnelle, la dramatisation temporelle et spéculaire, l'adresse " vive ». On observe aussi que la radiophonie exacerbe de manière théâtrale des dimensions poétiques : la résonance, la rencontre sonore d'une altérité transsubjective, la transmission d'expérience (spéculative narrative). Poéticité, théâtralité et narrativité se conjuguent ainsi dans la pièce de manière complémentaire. J'aimerais désormais revenir sur deux autres pièces radiophoniques au sein desquelles ces diverses dimensions sont également à l'œuvre : Urban Prayers de Björn Bicker ${ }^{23}$

21. - Les réflexions sur l'être-ensemble infusent toute l'œuvre du philosophe. Ses maîtres ouvrages en la matière sont: Jean-Luc Nancy, La communauté désœuvrée, Paris, Christian Bourgois, 1986, et Être singulier-pluriel, Paris, Galilée, 1996.

22. - Hartmut Rosa, Résonance: une sociologie de la relation au monde, Paris, La Découverte, 2018. Je me rapporte à l'édition allemande: H. Rosa, Resonanz : Eine Soziologie der Weltbeziehung, Frankfurt a.M., Suhrkamp, 2016, p. 336.

23. - Urban Prayers de Björn Bicker, production BR2, 2014. Ce Hörspiel est issu d'un spectacle cette fois (présenté dans des églises) et non d'un poème : c'est pourquoi la pièce radiophonique est produite en partenariat avec les Münchner Kammerspiele, 
et Europas längster Sommer de Maxi Obexer ${ }^{24}$. En vertu de contenus plus prosaïques, la première met davantage l'accent sur la théâtralité, la seconde sur la narrativité. Mais chaque pièce est matricée par ces dynamiques poétiques-théâtrales de la poésie sonore, et j'aimerais voir comment chacune est ainsi apte à devenir un lieu de passage, voire de transmission, d'une expérience.

\section{Au-delà de transsubjectivité le rythme de voix plurielles}

Urban Prayers présente de nombreuses qualités formelles très semblables à Ewig ohne Dauer. Le texte est à nouveau porté par trois acteurs ${ }^{25}$. Les voix alternent de façon régulière, et mettent en exergue le caractère très rythmé du texte. Celui-ci est presque de part en part traversé par l'anaphore « et que pensez-vous donc ? $\gg^{26}$. Des questions à l'auditeur s'enchaînent sans attendre de réponse : que pensez-vous que nous mangeons ? que nous pensons ? à quoi nous acquiesçons ? Ce très fort dialogisme est d'emblée transsubjectif : les questions traitent des divers modes de vie à l'intérieur de nos vies multiculturelles et prêtent à réfléchir sur le concept même de multiculturalité, de démocratie plurielle et/ou agonistique. Elles sont certes beaucoup plus éloignées de l'expression d'un « je lyrique ». En revanche, le texte accumule d'autant plus de variations sur un même thème, ou sur une même forme langagière : la répétition d'expressions rythme ainsi très fortement le texte, en sus de l'anaphore interpellative. Des listes d'adjectifs qualificatifs très imagés font suite à une énumération de lieux, ou de supports pour dormir par exemple (des matelas, des pailles, des chaises, des pierres, des bancs), ce qui affecte les mots d'une dimension très sensible. Des phrases affirmatives succèdent à des phrases négatives, ni négatives, ni affirmatives ( $«$ je dis oui. Non. Je ne dis ni oui, ni non ${ }^{27} »$, «Pensez-vous que nous donnons la main ? que nous ne donnons pas la main ? $\left.{ }^{28}\right)$ : ces revirements donnent lieu à des retournements de pensée encore plus performatifs à l'écoute qu'à la lecture (plus anticipatrice).

et énoncée par des acteurs du grand théâtre munichois. Abrégé par la suite par Bicker et indications des minutes.

24. - Europas längster Sommer de Maxi Obexer, produit par WDR 3, 2018. Les voix appartiennent à Margareth Obexer, Glenn Goltz, et Katharina Schmalenberg. La pièce est disponible en podcast. Voir https://www.youtube.com/watch? $\mathrm{v}=\mathrm{d} 2 \mathrm{Ih} 9 \mathrm{OfpLi8}$, consulté le 25 mai 2019. Abrégé par la suite par Obexer et indications des minutes.

25. - Wiebke Puls, Edmund Telgenkämpfer, Cigdem Teke.

26. - La pièce commence ainsi : "Was denkt ihr denn? " appliqué à divers domaines : « Was denkt ihr denn, was wir tun? was wir essen ? wo wir wohnen ? ...». Les anaphores sont particulièrement récurrentes durant les cinq premières minutes.

27. - " Ich sage ja. nein. Ich sage weder ja noch nein » (Bicker, 12’10).

28. - « Was denkt ihr denn, warum wir die Hand reichen? Warum wir die Hand nicht reichen? » (ibid., 10'15). 
Plus encore qu'Ewig ohne Dauer, le texte composé de listes se rapproche des pièces-paysages de Gertrude Stein. Il passe d'autre part par des moments de réflexion thématique clairement articulés : les modes de rencontre, les habitats, les besoins de lieux de prières, la réaction en hôpital, vis à vis du sport, etc. Ces moments développent, comme le laisse entendre l'étymologie scientifique du vocable moment, chacun une dynamique rythmique propre. Les propos très denses sont par ailleurs ponctués par des pauses, musicales, meublées par des bits et des accords électroniques. La musique est souvent plus chantée, plus narrative, plus sophistiquée que dans Ewig ohne Dauer, son empreinte est donc plus marquée, le texte en contrepoint en devient également plus musical.

À la différence d'Ewig ohne Dauer, la pluralité des voix répond cette fois à une pluralité extrême des dires. L'auteur s'est appuyé sur plusieurs centaines de témoignages d'habitants de Munich, récoltés pendant deux ans. De la sorte, la diversité des assertions rend la multiplicité des voix palpable, elle renvoie à des voix différentes et non seulement à une pensée plurielle, susceptible de ressortir d'un être singulier-pluriel. La multiplicité va cependant de pair avec une composition claire, un espace radiophonique uni, rassemblant tous ces dires au sein d'un même format. Comme les sujets ne sont pas identifiables à travers les voix des acteurs, la polyphonie anonyme devient allégorique, quand la pièce traite des conditions de vie prosaïques, relatives aux modes de vie urbains dans les métropoles contemporaines, et aux communautés diverses qui y vivent. S'ensuivent deux conséquences remarquables.

D'une part, les conditions de vie évoquées dressent des « scènes de la pensée » beaucoup plus imagées et théâtrales qu'Ewig ohne Dauer. Mais d'autre part, l'enjeu reste spéculatif. En effet, la pièce traite de questions philosophiques et culturelles par excellence, puisqu'elle interroge les croyances contemporaines (comme l'indique son titre, Urban prayers). Ces croyances et convictions sont redevables à des religions, mais aussi à des habitus culturels. Or les habitus sont souvent avancés sans être réfléchis plus avant mais leur extrême diversité, jointe à leur accumulation et leur juxtaposition, montrent qu'ils sont loin de pouvoir être naturalisés. L'accumulation les relativise, et montre leur constitution de vecteur identitaire, culturel, formant autant de barrières aléatoires selon le lieu et l'époque de naissance de la personne interrogée. La pièce constitue in fine une réflexion sur l'identité et la culture au-delà de toute expression émotionnelle, a fortiori de crispation. Néanmoins les situations décrites contribuent à développer l'imaginaire des auditeurs, leur engagement subjectif, voire autopoḯtique. Le poème ne transmet pas des informations, mais transmet des expériences dispersées, fractalisées. Ainsi par ses fortes dimensions imagée et spéculative, particulièrement sensibles en vertu du rythme global et de la déconnection de dires singuliers et situés, il est apte à transmettre une expérience qui touche tout habitant 
des villes, sans buter sur des obstacles identitaires ou émotionnels. Sa réflexion s'appuie par ailleurs encore une fois sur le langage : le terme même de croyance est approché d'une multitude de points de vue, et dans les comportements les plus rationnels des concitoyens démocratiques se nichent des idéologies non réfléchies, des éléments de crispation malgré eux ; mais ils sont pratiquement sublimés.

À la différence d'Ewig ohne Dauer, la pièce a une dimension politique, mais ne transmet pas de position idéologique. Les dissensions sur les habitus, la tolérance manifestée ou non, le partage de la ville et les délimitations de territoires, les négociations abouties ou en échec, touchent cependant le partage du sensible, et de manière très directe ${ }^{29}$ : ce partage est en jeu du point de vue des pratiques, et du point de vue des concepts et négociations qui les sous-tendent. Le questionnement du partage sensible n'est d'ailleurs pas absent d'Ewig ohne Dauer : la pièce met également en cause les modes de reconnaissance, en l'occurrence l'absence de reconnaissance du rêve, de sa nécessité pour la pensée au même titre que le désir. Or questionner la reconnaissance, c'est interroger les modes de subjectivation contemporains qui doivent l'accompagner. Voilà encore un élément narratif important de la pièce. Dans Urban Prayers, les multiples perspectives singulières veulent toutes être reconnues. Or l'espace de la pièce est ce moyen de reconnaissance, il représente un seul espace sensible, qui rassemble les différences. Grâce à la qualité poétique du langage, il invite à transcender les différences dans un même rythme, une écoute égale, une attention de tous les instants. Il est même susceptible de promouvoir une telle attitude. Il permet de passer outre l'étrangeté des habitus, et parfois des voix, des accents, pour développer une appréhension tant plurielle que nouvelle en ce qu'elle subsume la pluralité des expériences de manière harmonieuse et quasi-utopique. Sans doute, le point de vue qui rallierait tous les points de vue est absent, mais la pièce est malgré tout à même, grâce à la poésie, de transmettre l'horizon du rassemblement, qui est peut-être nécessaire en démocratie.

\section{D'un lyrisme narratif porté par une seule voix}

J'aimerais enfin me pencher sur un dernier exemple de pièce, beaucoup moins collaborative, beaucoup plus linéaire et narrative, et qui s'appuie sur une seule voix radiophonique. Il me semble intéressant de voir comment une narration fondée sur une forme moins poétique, peut néanmoins, en s'appuyant sur des dynamiques théâtrales radiophoniques, devenir une spéculation poétique apte à transmettre une expérience. La

29. - Cette expression a évidemment trait à l'ouvrage sur l'esthétique de la politique de Jacques Rancière, Le Partage du sensible, Paris, La Fabrique, 2000. 
narratrice d'Europas längster Sommer est son autrice, comme dans Ewig ohne Dauer. À l'instar des deux Hörspiele précédents, sa pièce repose d'abord sur un questionnement incessant. Dès le début, elle demande qu'est-ce qu'arriver en Europe ? Où est ce pays qui n'existe pas ? L'autrice se décrit comme migrante, et retrace la route qu'elle a suivie du Tyrol vers l'Allemagne ; elle revient sur les circonvolutions entre l'être étranger et non-étranger, le chemin que l'on fait et les lieux où l'on arrive, la langue qui nous est propre ou le propre de la langue.

Bien que narrative, la pièce est ici encore d'abord soumise au pouvoir des mots et des imaginaires disruptifs. Pareils à des nexus liant passé, présent et futur, les mots constituent les principaux vecteurs d'expérience. L'autrice revient sur ceux qui lui sont chers : «befristete Aufenthaltserlaubnis », « Freizügigkeitsbescheinigung », « Ausländer Einwohner Meldeamt », « Integration », « Einwanderung », « Heimat », «Zugehörigkeit ». Elle rêve de la reconnaissance d'être une étrangère comme les autres : à ces réflexions nostalgiques se mêle beaucoup d'humour, mais elle souligne par ses considérations le caractère hautement symbolique des termes administratifs banalisés, caractère qui échappe aux citoyens, et dans une certaine mesure, aux personnels administratifs. La puissance de nomination recouvre encore une puissance affective. Les mots donnent par ailleurs lieu à des associations, des histoires fragmentaires : personnes rencontrées, voyages ou démarches effectués. Certaines anecdotes sont aphoristiques ou métaphoriques : rituel d'adieu de la mère, invention d'un nouveau rituel, première personne dont la patrie est la langue, jeunes garçons dans le train, un futur qui s'ébauche, et quelques minutes plus tard, leur arrestation. Le récit enfile les incongruités comme des perles, le voyage vers l'administration, les froideurs des lieux et des accueils se combinent à la familiarité de la langue jouissant de toutes les opportunités culturelles. Une pensée subtile, nourrie de contradictions et de la jouissance des éclats de la langue, se déplie, ressemblant en cela à des images de pensée benjaminiennes, des poèmes en prose subsumant toutes les incongruités. Le rythme, ici encore, est cardinal. L'intonation est égale, presqu'atone. Elle introduit une rupture profonde avec les formats standardisés des documentaires, rupture qui s'ajoute au travail stylistique, métaphorique et disruptif. Le rythme achève de faire du texte un poème qui n'est pas passeur d'informations mais d'expérience et de spéculation.

Lorsqu'enfin se dessine la biographie de l'autrice, celle-ci pointe avec ironie la dimension de son errance, de ce voyage initiatique en un pays qui doit devenir le sien et où « elle se trouverait». Elle s'amuse de sa Wanderung peu romantique, ses considérations sont légères sur un parcours qui est lourd, d'autant que ladite Wanderung rime avec Einwanderung et Auswanderung. Elle se met en écho de celle de millions d'autres personnes qui ont délaissé leur pays, et d'une réflexion 
sur l'appréhension, le décalage, l'accueil et le sentiment d'être arrivé. Or ce questionnement des termes recouvre toujours une mise en cause du réel, des catégories établies comme autant de barrières axiomatiques et existentielles non seulement pour ce qui la concerne mais pour des millions d'autres, des personnes d'origines, de statuts, d'habitus différents, qui n'ont peut-être pas les mêmes motivations de partir, mais qui partagent « le fait d'être parties pour arriver quelque part ${ }^{30}$. Nul chœur, nulle liste dans ce texte, presqu'aucune répétition de phrases avec variations ${ }^{31}$, mais toute la narration est transsubjective, et par ailleurs suspendue : suspendue au désir de trouver un pays, une langue, suspendue à une spéculation sur l'étrangeté, sur sa propre altérité, et sur une altérité partagée avec d'autres étrangers. Ces éléments sont répétés et remodulés, si ce n'est que la narratrice vise un pays bien précis, et une langue qui est déjà la sienne, l'allemand ${ }^{32}$. Elle redéfinit les termes des étrangetés possibles, et contraint l'auditeur à des retournements de pensée incessants. Pour que finalement, l'arrivée dans des mots, dans des usages, dans des imaginaires soit considérée d'égale et essentielle importance pour tous, que la spéculation repose sur les modulations de ce que « arriver » ou « patrie » peuvent vouloir dire bien au-delà de significations littérales. Même dans la deuxième partie plus narrative, la transmission d'expérience s'appuie comme dans Ewig ohne Dauer sur la puissance de résonance, tant émotionnelle, langagière qu'imaginaire.

Notons qu'au fur et à mesure de la pièce, la pièce devient plus narrative que réflexive. Mais le voyage garde une dimension allégorique jusque dans la deuxième partie, car l'autrice est partie du Tyrol montagnard, où on n'usait de la parole qu'en dernier recours, pour se mettre en quête d'un pays où elle pouvait user de la langue. Pendant la première moitié de cette pièce assez brève (une demi-heure au total), les allers et retours de la réflexion sont à l'image du parcours, à la fois dynamique et statique de l'autrice (dans une même langue et culture). Ils sont également susceptibles de faire écho à l'auditeur de manière performative : tout comme la narratrice, l'auditeur de radio est à la fois « dans » la langue qu'il entend et au dehors, dans le lieu de la parole sans qu'elle lui appartienne néanmoins, dans un endroit en même temps familier et rendu théâtral par l'écoute. Il est susceptible d'éprouver un écho situationnel, y compris en réalisant la profondeur des dimensions immatérielles d'une situation, d'une appartenance à

30. - «weg gegangen um anzukommen » (Obexer, 26’47).

31. - On note surtout les jeux de mots très rythmés et répétés sur « frei zügig sein/ sei frei zügig » (ibid., 3’32).

32. - Un exemple « d'image de pensée » parmi d'autres est la mention de l'avidité qui s'éprend des mots, laquelle conduirait la narratrice à surcharger les tables de feuilles au point que les pieds défoncent le plancher. 
un pays ou une langue. Les dramatisations temporelles, spéculaires, spéculatives, sont ici ostensibles, d'autant que le drame intersubjectif et transsubjectif de l'arrivée en pays étranger est étendu à tous « les gens du voyage » ou, plus précisément, de la migration. Le caractère poétique du texte est donc intimement lié à une spéculation sur l'être-dans-la-langue, le désir de langue, le fait d'être en errance, en immigration, émigration et migration. Quelques jeux de mots et de pensée sous-tendent la réflexion : " et ce qui migre en nous jour après jour a de plus en plus de poids » (« es wird immer schwerer, was [Tag für Tag] in einen hineinwandert », Obexer, 6'13).

La pièce livre d'autres bases d'inscription situationnelle, de dramatisation temporelle et spéculaire, de résonance, de rencontre inter- et transsubjective, que les précédentes, mais là encore elle ouvre un espace poétique-théâtral d'expérience susceptible de toucher l'auditeur : celui-ci peut mettre en résonance ses propres expériences d'éet d'immigration, plonger dans les mots pour donner un nom à ses expériences, et arriver à les saisir à l'intérieur de sociétés et d'espaces médiatiques qui ne donnent guère l'occasion d'investir subjectivement ces mots-clés qui nous déterminent par le biais du dire poétique. 